\title{
Matrix analysis techniques in cage induction machines
}

\author{
David G. Dorrell \\ Department of Electronics and Electrical Engineering, \\ University of Glasgow, Glasgow, UK \\ E-mail: d.dorrell@elec.gla.ac.uk
}

\begin{abstract}
Modern analysis techniques for electrical machines are either analytical or use finite element analysis. Both methods are implemented using computational techniques to solve the magnetic circuit and produce performance predictions. This paper puts forward a steady-state impedance matrix method for analysing a split-phase induction machine that is at the leading edge of analytical modelling of induction motors. The method is implemented and verified against an example to illustrate the asynchronous torques in a non-sinusoidal winding. In the second half of the paper it is shown how the technique can be applied to a three-phase machine and the classical per-phase model is obtained from the derivation. The steady-state model derives a mathematical rigorous equivalent circuit that includes the skew reactance term in the rotor circuit.
\end{abstract}

Keywords: split phase induction motors; 3-phase induction motors; impedance matrix

Reference to this paper should be made as follows: Dorrell, D. G. (2008) 'Matrix analysis techniques in cage induction machines', Int. J. Computer Applications in Technology, Vol.

\section{INTRODUCTION}

The circuits and cross-coupling in an induction machine can be solved using an impedance matrix where, for an applied voltage, the currents are resolved and the torque obtained under steady-state operation. Williamson and Smith (1982) developed a model which utilizes an impedance matrix using the conductor density method. This has been further modified to investigate winding shorts [Williamson and Mirzoian (1985)], broken bars [Williamson, Abdel-Magied and Smith (1987)], rotor eccentricity [Dorrell and Smith (1996)] and inter-bar current [Dorrell, Miller and Rasmussan (2003)], amongst other things. The method has also been used to analyse brushless doubly-fed machines of the induction type [Williamson, Ferreira and Wallace (1997)] and reluctance type [Dorrell (2007)]. All these papers are couched in terms of 3-phase induction machines. It can also be used for single phase and split-phase induction machines [Dorrell and Jensen (2007)] and these are the focus points of this paper.

This paper will address two issues that have not been reported in the literature to any great extent. It will firstly put forward representations for a split phase induction motor where the auxiliary and main winding connections are considered separately. This allows the auxiliary winding to be connected via a start/run capacitor or resistor. It also includes MMF harmonics so that the asynchronous torque dips due to the $3^{\text {rd }}, 5^{\text {th }}, 7^{\text {th }}$, etc. spatial MMF harmonics are present in the torque/speed curves. The matrix analysis also allows any combination of unbalanced winding. The second part of the paper will show how this method does produce the standard per-phase model for the machine and illustrate the equivalent circuit parameters in terms of the matrix impedances. The methods are programmed in the MATLAB environment to illustrate the implementation of the transient model.

\section{CONDUCTION DENSITY METHOD FOR SPLIT PHASE MACHINE}

This section will outline the conduction density method and the derivations of the air-gap flux waves and electric field equations. It will then go on to develop the impedance matrix and solve for the torque. The method is applied to a split phase machine which are generally sub-horsepower machines and extensively applied to pumping and refrigeration applications.

\subsection{Stator and rotor conductor representation}

Split-phase induction motors are usually 2-pole machines with a main and an orthogonal auxiliary winding. The main 2-pole winding coefficients can be defined by the general equation: 


$$
\begin{aligned}
\bar{N}_{\text {main }}^{n} & =\frac{1}{2 \pi r} \sum_{W=1}^{\text {slot no }} \frac{\sin \left(\frac{p n k b_{s}}{2}\right)}{p n k b_{s} / 2} C_{w} e^{j n \theta_{w}} \\
& =\frac{1}{2 \pi r} \sum_{W=1}^{\text {slot no }} k_{s}^{n} C_{w} e^{j n k y_{w}}
\end{aligned}
$$

where $C_{w}$ is the number of conductors in the slot, $y_{w}$ is the linear circumferential location of the centre of the slot, $\theta_{w}$ is the angular location of the slot (in mechanical degrees), $r$ is the mean air-gap radius, $k$ is the inverse of $r, p$ is the machine pole-pair and $n$ is the winding harmonic. The slot opening factor is given by $k_{s}^{n}$ and $b_{s}$ is the slot opening. A similar equation can be obtained for the auxiliary winding. In a split phase machine we need to allow for the grading of the winding, i.e., the coils to have different numbers of turns. This reduces the spatial harmonic content of the winding. As an example of the implementation of (1), the 2pole winding in Figure 1 (where $C_{n}$ is the number of coilturns in the slot) is:

$$
\begin{aligned}
\bar{N}_{\text {main }}^{n}= & \frac{k_{s}^{n}}{2 \pi r} \times \\
& {\left[\begin{array}{l}
C_{2} e^{j n \frac{1.5}{24} \pi}+C_{3} e^{j 3 n \frac{2.5}{24} \pi}+C_{4} e^{j n \frac{3.5}{24} \pi}+C_{5} e^{j n \frac{4.5}{24} \pi} \\
+C_{6} e^{j n \frac{5.5}{24} \pi}+C_{7} e^{j n \frac{6.5}{24} \pi}+C_{8} e^{j 3 n \frac{7.5}{24} \pi}+C_{9} e^{j n \frac{8.5}{24} \pi} \\
+C_{10} e^{j n \frac{9.5}{24} \pi}+C_{11} e^{j n \frac{10.5}{24} \pi}-C_{14} e^{j n \frac{13.5}{18} \pi}-C_{15} e^{j n \frac{14.5}{18} \pi} \\
-C_{16} e^{j n \frac{15.5}{18} \pi}-C_{17} e^{j n \frac{15.5}{18} \pi}-C_{18} e^{j n \frac{16.5}{18} \pi}-C_{19} e^{j n \frac{17.5}{18} \pi} \\
-C_{20} e^{j n \frac{18.5}{18} \pi}-C_{21} e^{j n \frac{19.5}{18} \pi}-C_{22} e^{j n \frac{20.5}{18} \pi}-C_{23} e^{j n \frac{21.5}{18} \pi}
\end{array}\right] }
\end{aligned}
$$

The rotor can be represented as a set of parallel loops formed from two adjacent bars and the connecting endrings. Therefore the number of loops is equal to the number bars. The harmonic current in each loop will be the same except for a phase shift. If the axial coordinate is $x$ (with centre zero - see Figure 1) and the angle of the skew is $\delta$ then the rotor loop distribution of two bars either side of the circumferential coordinate $y=0$ is

$\bar{n}_{r}^{n}(x, y)=\bar{N}_{r}^{n} e^{-j n k y_{b a r}} e^{-j n k x \tan \delta}$

where

$$
\bar{N}_{r}^{n}=-j \frac{k_{r}^{n}}{\pi r} \sin \left(\frac{n \pi}{N_{b}}\right)
$$

$k$ is the inverse of the mean air-gap radius $r, N_{b}$ is the number of rotor cage bars and $k_{r}^{n}$ is the rotor slot opening (unity for closed slot). We can also define a skew factor:

$$
k_{s k}^{n}=\frac{2 \sin \left(\frac{n k l \tan \delta}{2}\right)}{n k l \tan \delta}
$$

These equations can be used to define the stator main and auxiliary spatial winding distributions together with the rotor cage spatial distribution.

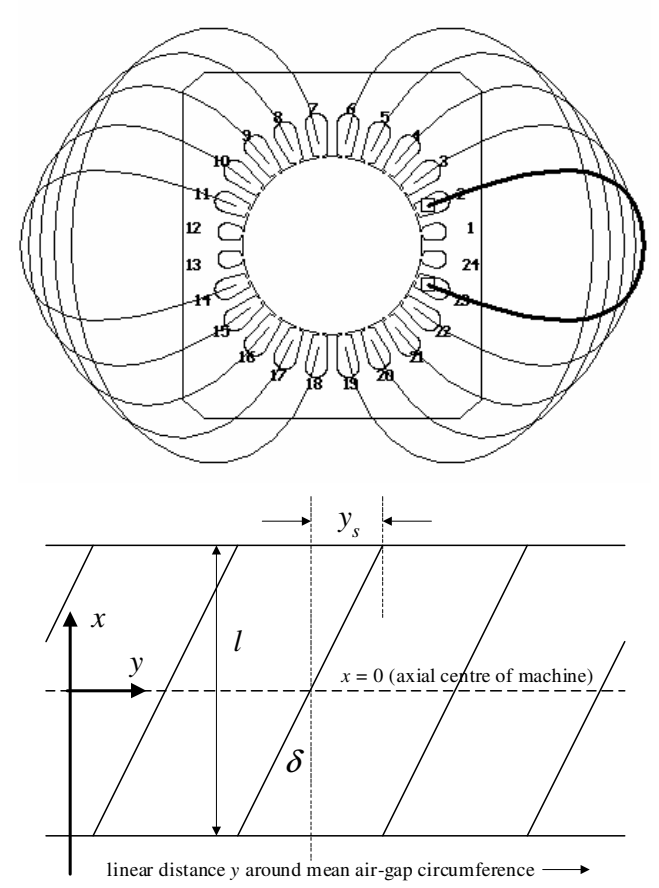

Figure 1 Main winding distribution and linearised rotor skew angle in the $x$ (axial) and $y$ (circumferential) directions

\subsection{Surface current density, air-gap fields and induced EMF}

The surface current density due to one winding is $j_{x}(y, t)=\operatorname{Re}\left[\sum_{-n}^{n} \bar{N}_{x}^{n} \bar{I}_{x} e^{j(\omega t-n k y)}\right]$

where $\omega$ is the supply frequency (in $\mathrm{rad} / \mathrm{s}$ ), $t$ is the point in time and $\bar{I}_{x}$ is the rms phasor of the winding current. We can use this to obtain the air-gap flux density where $b(y, t)=\frac{\mu_{0}}{g} \int j_{s}(y, t) d y$

The electric field strength from a one-dimensional Curl function can be found from

$e(y, t)=\int \frac{d b(y, t)}{d t} d y$

If we have another winding defined in a similar way then we can obtain the voltage induced into the winding, and hence obtain the impedance from:

$$
\begin{aligned}
u_{y}(t) & =\operatorname{Re}\left[\int_{0}^{2 \pi}-e(y, t) n_{y}(y) l \cdot d y\right] \\
& =\operatorname{Re}\left[j X_{x y} \bar{I}_{x} e^{j \omega t}\right]
\end{aligned}
$$

The rotor is treated as a series of parallel circuits of adjacent bars with a phase offset. Only the harmonic currents in one rotor loop needs to be obtained if symmetry is present. Skew can also be included by use of an axial $(x)$ variation and (5). A full derivation of this can be found in Williamson and Smith (1982) and given later. It is relatively easy to apply. Here it is applied to low voltage parallel main windings in a split phase induction motor. 


\subsection{Circuit and impedance matrix}

In this connection the main winding is connected in parallel with the auxiliary winding. This is shown in Fig. 2. Several MMF harmonics are included in the model, hence it accounts for asynchronous torques dips. The matrix for the parallel-connected impedance matrix is

$\left[\begin{array}{l}\bar{V}_{p h} \\ \bar{V}_{p h} \\ 0 \\ \ldots \\ 0 \\ 0 \\ \ldots \\ 0 \\ \bar{V}_{p h} \\ \bar{V}_{p h}\end{array}\right]=\left[\begin{array}{ccccccccccccc}\bar{Z}_{m} & 0 & \bar{Z}_{r m}^{23} & \ldots & \bar{Z}_{r m}^{3} & \bar{Z}_{r m}^{1} & \bar{Z}_{r m}^{-1} & \bar{Z}_{r m}^{-3} & \ldots & \bar{Z}_{r m}^{-23} & \bar{Z}_{C m m} & 0 \\ 0 & \bar{Z}_{a} & \bar{Z}_{r a}^{23} & \ldots & \bar{Z}_{r a}^{3} & \bar{Z}_{r a}^{1} & \bar{Z}_{r a}^{-1} & \bar{Z}_{r a}^{-3} & \ldots & \bar{Z}_{r a}^{-23} & 0 & \bar{Z}_{C a a} \\ \bar{Z}_{m r}^{23} & \bar{Z}_{a r}^{23} & \bar{Z}_{r r}^{23} & \ldots & 0 & 0 & 0 & 0 & \ldots & 0 & 0 & 0 \\ \ldots & \ldots & \ldots & \ldots & \ldots & \ldots & \ldots & \ldots & \ldots & \ldots & \ldots & \ldots \\ \bar{Z}_{m r}^{1} & \bar{Z}_{a r}^{1} & 0 & \ldots & 0 & \bar{Z}_{r r}^{1} & 0 & 0 & \ldots & 0 & 0 & 0 \\ \bar{Z}_{m r}^{-1} & \bar{Z}_{a r}^{-1} & 0 & \ldots & 0 & 0 & \bar{Z}_{r r}^{-1} & 0 & \ldots & 0 & 0 & 0 \\ \ldots & \ldots & \ldots & \ldots & \ldots & \ldots & \ldots & \ldots & \ldots & \ldots & \ldots & \ldots \\ \bar{Z}_{m r}^{-23} & \bar{Z}_{r a}^{-23} & 0 & \ldots & 0 & 0 & 0 & 0 & \ldots & \bar{Z}_{r r}^{-23} & 0 & 0 \\ \bar{Z}_{m C m} & 0 & 0 & \ldots & 0 & 0 & 0 & 0 & \ldots & 0 & \bar{Z}_{C m} & 0 \\ 0 & \bar{Z}_{a C a} & 0 & \ldots & 0 & 0 & 0 & 0 & \ldots & 0 & 0 & \bar{Z}_{C a}\end{array}\right]\left[\begin{array}{l}\bar{I}_{m} \\ \bar{I}_{a} \\ \bar{I}_{r}^{23} \\ \ldots \\ \bar{I}_{r}^{1} \\ \bar{I}_{r}^{-1} \\ \ldots \\ \bar{I}_{r}^{-23} \\ \bar{I}_{C m} \\ \bar{I}_{C a}\end{array}\right]$

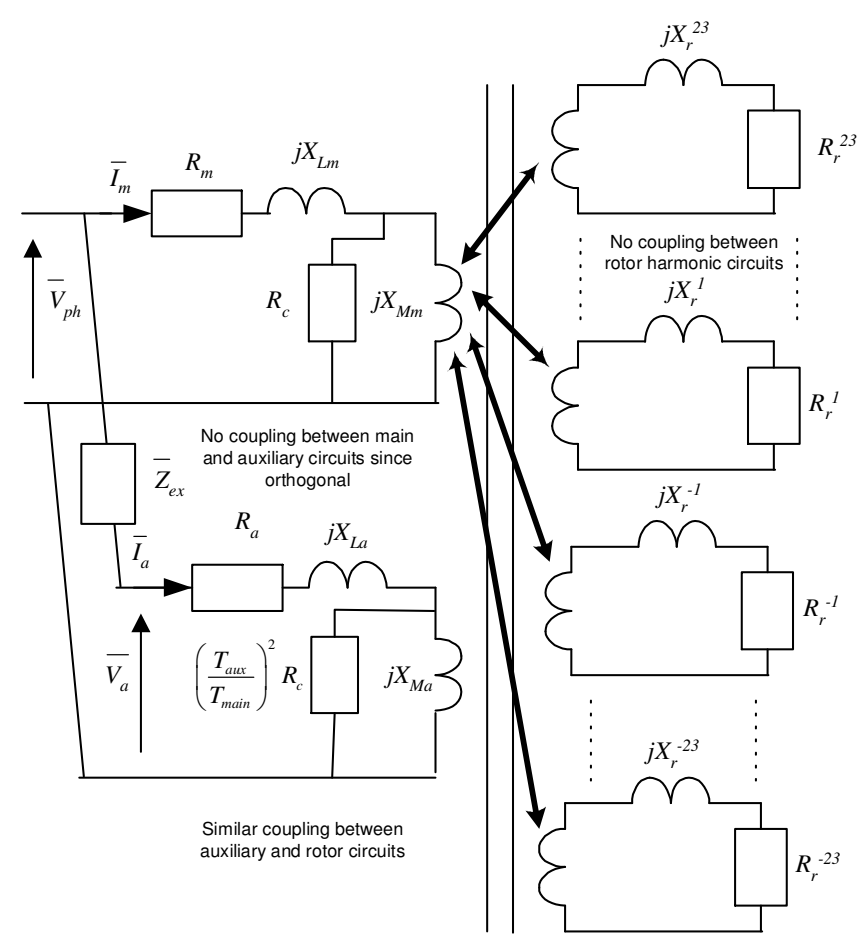

Figure 2 Split-phase machine circuit including higher winding harmonics

The first two rows represent the voltage equations for the main and auxiliary windings respectively while the final two rows are the voltage equations round the core-loss resistances of the equivalent circuit as illustrated in Figure 2. The remaining rows represent each rotor MMF harmonic. These are spatial harmonics (denoted by the index). For each spatial harmonic there is a forwards and backwards rotating flux wave and this is indicated by the positive and negative indexes. In a balanced 3-phase machine only the 1 ,
TABLE I. WINDING LAYOUT MACHINE WITH
HIGH MMF CONTENT IN MAIN AND AUXILIARY WINDING

\begin{tabular}{|c|c|c|}
\hline Slot no. & $\begin{array}{c}\text { Main } \\
\text { Winding } \\
{[\%]}\end{array}$ & $\begin{array}{c}\text { Auxiliary } \\
\text { Winding [\%] }\end{array}$ \\
\hline 1 & 0 & 5.8 \\
\hline 2 & 0 & 22.1 \\
\hline 3 & 7.8 & 22.1 \\
\hline 4 & 8.6 & 0 \\
\hline 5 & 17.6 & 0 \\
\hline 6 & 16 & 0 \\
\hline 7 & 16 & 0 \\
\hline 8 & 17.6 & 0 \\
\hline 9 & 8.6 & 0 \\
\hline 10 & 7.8 & 22.1 \\
\hline 11 & 0 & 22.1 \\
\hline 12 & 0 & 5.8 \\
\hline & & \\
\hline
\end{tabular}

$-5,7,-11,13, \ldots$ harmonics exist however in a single phase or split phase machine there is a full set of forwards and backwards MMF harmonics including third harmonics.

The main winding impedance consists of the winding resistance, the leakage inductance and the main inductance (which produces air-gap flux), which contains the MMF spatial harmonics. The auxiliary winding is similar, with the addition of the external impedance (necessary to produce a phase shift of the voltage at the auxiliary winding terminals). The impedances are given in the next section. The winding layouts of the test machine are given in Table I. This is designed to give high asynchronous torques to highlight the method.

\subsection{Impedance components - stator-stator impedances}

The main winding self-impedance is

$$
\bar{Z}_{m}=R_{m}+j X_{M m}+j X_{L m}
$$

where $R_{m}$ is the winding resistance and $X_{L m}$ is the total of the slot, differential and end-winding leakage (which can be obtained from SPEED, Miller (2006) and other methods).

The main winding reactance is

$X_{M m}=X_{\text {coef }} \sum_{n} \frac{\bar{N}_{\text {main }}^{n} \bar{N}_{\text {main }}^{n^{*}}}{n^{2}}$

The auxiliary winding has a similar equation

$\bar{Z}_{a}=R_{a}+j X_{M a}+j X_{L a}+\bar{Z}_{e x}$

where auxiliary winding resistance and leakage can be obtained from SPEED and $\bar{Z}_{e x}$ is the external impedance which could be a capacitance or start resistance.

The auxiliary winding self-impedance is

$X_{M a}=X_{c o e f} \sum_{n} \frac{\bar{N}_{a u x}^{n} \bar{N}_{a u x}^{n^{*}}}{n^{2}}$

The core loss self-impedances are:

$\bar{Z}_{C m}=R_{m}+R_{c}+j X_{L m}$

and

$\bar{Z}_{C a}=R_{a}+T_{\text {ratio }}^{2} R_{c}+j X_{L a}+\bar{Z}_{e x}$ 
There are also mutual impedances between these components:

$$
\begin{aligned}
& \bar{Z}_{C m m}=\bar{Z}_{m C m}=R_{m}+j X_{L m} \\
& \text { and } \\
& \bar{Z}_{C a a}=\bar{Z}_{a C a}=R_{a}+j X_{L a}+\bar{Z}_{e x}
\end{aligned}
$$

\subsection{Impedance components - rotor-rotor impedances}

The self impedances of one loop take the form of:

$$
\begin{aligned}
\bar{Z}_{r r}^{n}= & 2\left(1-\cos \left(n \theta_{b}\right)\right)\left(R_{b}^{n}+j X_{\text {slot }}^{n}\right) \\
& +2 R_{\text {end-ring }}+j 2 X_{\text {end-ring }}+j\left(X_{r}^{n}+X_{\text {diff }}^{n}\right)
\end{aligned}
$$

where the effective bar resistance is

$R_{b}^{n}=k_{s k i n}^{n} \times$ Bar resistance

and $k_{\text {skin }}^{n}$ is a skin effect resistance coefficient. The end-ring is represented by segments which connect adjacent bars and are denoted by $R_{\text {end-ring }}$. The end-ring reactance $X_{\text {end-ring }}$ is often neglected. The end-rings often have different crosssections and this can be incorporated here if necessary. The angle $\theta_{b}$ is the pitch of the rotor bars so that

$$
\theta_{b}=2 \pi / \text { Bar number }
$$

The main self inductance is defined by

$X_{r}^{n}=X_{\text {coef }} \times|1-n(1-s)| \times \frac{\bar{N}_{r}^{n} \bar{N}_{r}^{n^{*}}}{n^{2}} \times$ Bar Number

The slot reactances can be obtained from the slot permeance:

$X_{\text {slot }}^{n}=\mu_{0} \omega_{s} l_{\text {bar }}|1-n(1-s)| \times S_{\text {bar }}$

The bar permeance $S_{b a r}$ can be obtained from standard equations or, as in this case, from SPEED. The bar length is $l_{\text {bar }}$. The differential reactance is defined by

$X_{r}^{n}=X_{\text {coef }}|1-n(1-s)| \sum_{v} \frac{\bar{N}_{r}^{(n+v)} \bar{N}_{r}^{(n+v)^{*}}}{(v+n)^{2}} \times$ Bar Number

\subsection{Impedance components - stator-rotor impedances}

The rotor currents link the stator winding through the following equations. The rotor currents do not link the coreloss voltage equations. However

$\bar{Z}_{r m}^{n}=j X_{c o e f} k_{s k}^{n} \frac{\bar{N}_{\text {main }}^{n} \bar{N}_{r}^{n^{*}}}{n^{2}} \times$ Bar Number

and

$\bar{Z}_{r a}^{n}=j X_{c o e f} k_{s k}^{n} \frac{\bar{N}_{a u x}^{n} \bar{N}_{r}^{n^{*}}}{n^{2}} \times$ Bar Number

\subsection{Impedance components - rotor-stator impedances}

There are slip frequency terms in these equations:

$\bar{Z}_{m r}^{n}=j(1-n(1-s)) X_{\text {coef }} k_{s k}^{n} \frac{\bar{N}_{r}^{n} \bar{N}_{\text {main }}^{n^{*}}}{n^{2}}$

and

$\bar{Z}_{a r}^{n}=j(1-n(1-s)) X_{c o e f} k_{s k}^{n} \frac{\bar{N}_{r}^{n} \bar{N}_{a u x}^{n^{*}}}{n^{2}}$

\subsection{Torque and other parameters}

The auxiliary-to-main turns ratio is given by

$T_{\text {ratio }}=\frac{T_{\text {aux }}}{T_{\text {main }}}=\left|\frac{\bar{N}_{\text {aux }}^{1}}{\bar{N}_{\text {main }}^{1}}\right|$

We can also define another constant where

$X_{\text {coef }}=\frac{2 \pi \omega_{s} r^{3} k_{s t k} L_{s t k} \mu_{0}}{k_{C}^{r} k_{C}^{s} g}$

where $\omega_{s}$ is the supply frequency in $\mathrm{rad} / \mathrm{s}, k_{s t k} L_{s t k}$, is the stack length including a stacking factor and $k_{C}^{r} k_{C}^{s} g$ is the air-gap length including Carter factor corrections for the stator and rotor slots. The input power is the real part of the complex conjugate of the $V I$ product:

$P_{i n}=\operatorname{Re}[V(1) \times((I(1)+I(2)+I(27)+I(28)) *]$

The input power can be obtained for each rotor harmonic:

$$
\begin{aligned}
P_{\text {in_rotor }}(n)= & \operatorname{Re}\left[\bar{Z}_{\text {mat }}(1, n+2) \times \bar{I}(n+2) \times \bar{I}(1) *\right] \\
& +\operatorname{Re}\left[\bar{Z}_{\text {mat }}(2, n+2) \times I(n+2) \times \bar{I}(2) *\right]
\end{aligned}
$$

The losses in the rotor can be obtained from $I^{2} R$ losses in the rotor cage and hence the mechanical output power for each harmonic and torque can also calculated.

The torque for a 2-pole machine is

$T=\sum_{n} \frac{P_{\text {mech }}(n)}{\omega_{r}}$

$=\sum_{n} \frac{\left(\begin{array}{l}P_{\text {in_rotor }}(n) \\ -N_{b}|\bar{I}(n+2)|^{2}\left(\left(1-\cos \left(m \theta_{b}\right)\right) R_{b}^{n}+2 R_{\text {end-ring }}\right)\end{array}\right)}{(1-s) \omega_{s}}$

where $m$ is the rotor harmonic number $(=25-2 n$ for $(10))$ and $N_{b}$ is the number of rotor cage bars. . The per-unit slip is $s$. In (32) the input power may be positive or negative, depending on the harmonic and slip, leading to the usual set of asynchronous torquesfor the spatial MMF harmonics.

\section{SIMULATIONS}

Some windings can contain many MMF harmonics and these can affect the torque/speed curve. This is illustrated in Figure 3 for a machine with an unbalanced winding. Table I shows the percentage of winding in each slot. The main has a large $3^{\text {rd }}$ winding harmonic while the auxiliary has a large $5^{\text {th }}$ and noticeable $7^{\text {th }}$.

The machine has very different starting and running torque characteristics as can be seen by comparison of Figures 3 and 4. During the start mode a $25 \mathrm{ohm}$ resistor is in series with the auxiliary winding to produce increased torque. It can be observed that the torque characteristic contains the $3^{\text {rd }}$ harmonic from the main winding and the $5^{\text {th }}$ harmonic from the auxiliary winding. However the run mode torque characteristic only contains $3^{\text {rd }}$ harmonic from the main winding where the machine is single phase. These results are compared to SPEED with fundamental MMF consideration only. 
The code for these simulations was developed in the MATLAB environment. This makes the algorithm development very straight forward since the matrices are all complex and the matrix inversion and solution of the currents can be simply carried out using:

$$
I=((\text { Zmat })) \backslash \operatorname{conj}\left(V^{\prime}\right) \text {; }
$$

Care should be made that this is not either singular or illconditioned. Once the current has been obtained the power components and torques can be calculated.

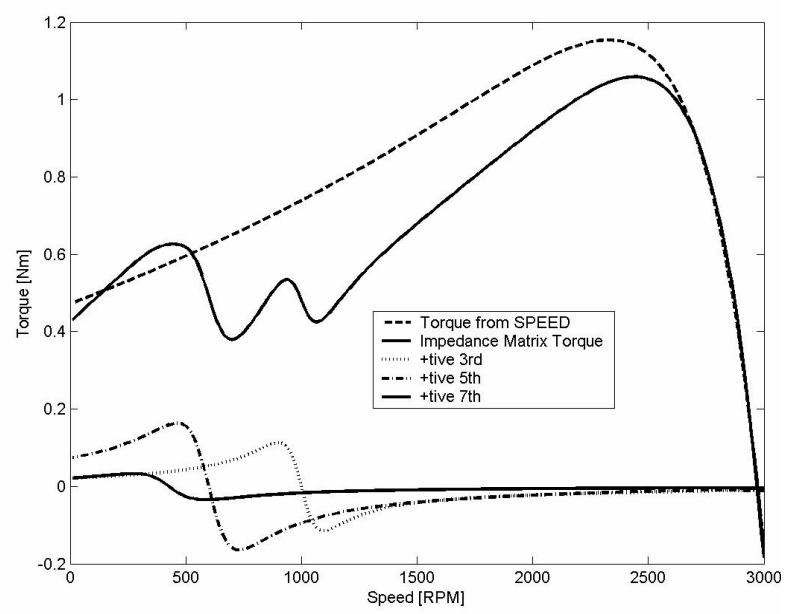

Figure 3 Torque-speed curves during starting

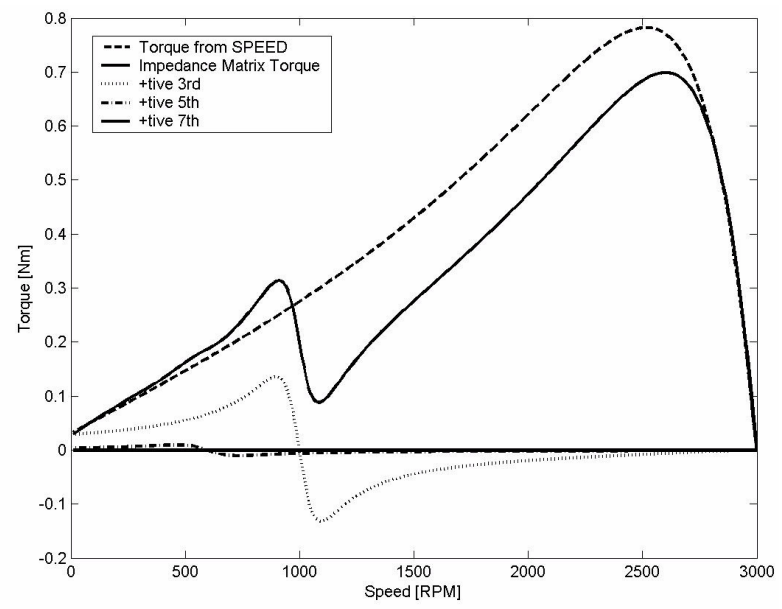

Figure 4 Torque-speed curves during run mode

\section{3-PHASE MACHINE REPRESENTATION - DERIVATION OF EQUIVELENT CIRCUIT}

In this section we will investigate the analysis of a 3-phase induction machine and derive the per-phase equivalent circuit. This will be couched in terms of the fundamental MMF wave and a balanced 3-phase winding and supply. First the winding is derived as a MMF spatial series to illustrate the harmonics that are obtained.

\subsection{3-phase winding MMF series}

Using (1) we can define phase $A$ where

$$
\bar{N}_{A}^{n}=\frac{1}{2 \pi r} \sum_{W=1}^{\text {slot no }} k_{s}^{n} C_{w} e^{j n k y_{w}}
$$

Where the total winding distribution is defined by

$n_{A}(y)=\sum_{n=1}^{\infty}\left(\bar{N}_{A}^{n} e^{-j n k y}+\bar{N}_{A}^{-n} e^{j n k y}\right)=\sum_{n=-\infty}^{\infty}\left(\bar{N}_{A}^{n} e^{-j n k y}\right)$

If we introduce the operator $a$ where

$a=e^{\frac{j 2 \pi}{3}}$

then for the $B$ and $C$ phases there are phase shifts in the three phase windings of $a^{n}$ and $a^{-n}$ so that

$$
\begin{aligned}
n_{B}(y) & =\sum_{n=1}^{\infty}\left(a^{n} \bar{N}_{A}^{n} e^{-j n k y}+a^{-n} \bar{N}_{A}^{-n} e^{j n k y}\right) \\
& =\sum_{n=-\infty}^{\infty}\left(a^{n} \bar{N}_{A}^{n} e^{-j n k y}\right)
\end{aligned}
$$

and

$n_{C}(y)=\sum_{n=-\infty}^{\infty}\left(a^{-n} \bar{N}_{A}^{n} e^{-j n k y}\right)$

where $\left.a^{n}\right|_{n=3 m}=1$ when $m=0,1,2$, etc.

For a balance 3-phase current set

$$
\begin{aligned}
& i_{A}(y, t)=\operatorname{Re}\left[\bar{I}_{A} e^{j \omega t}\right] \\
& i_{B}(y, t)=\operatorname{Re}\left[a^{-1} \bar{I}_{A} e^{j \omega t}\right] \\
& i_{C}(y, t)=\operatorname{Re}\left[a \bar{I}_{A} e^{j \omega t}\right]
\end{aligned}
$$

The stator surface MMF is then

$$
\begin{aligned}
\left.j_{s}(y, t)\right) & =n_{A}(y) i_{A}(y, t)+n_{B}(y) i_{B}(y, t) \\
& +n_{C}(y) i_{C}(y, t)
\end{aligned}
$$

By combining (35) and (37)-(38)

$$
j(y, t)=\operatorname{Re}\left[\begin{array}{l}
\sum_{n=-\infty}^{\infty}\left(\bar{N}_{A}^{n} \bar{I}_{A} e^{j(\omega t-n k y)}\right) \\
+\sum_{n=-\infty}^{\infty}\left(a^{n} \bar{N}_{A}^{n} a^{-1} \bar{I}_{A} e^{j(\omega t-n k y)}\right) \\
+\sum_{n=-\infty}^{\infty}\left(a^{-n} \bar{N}_{A}^{n} a \bar{I}_{A} e^{j(\omega t-n k y)}\right)
\end{array}\right]
$$

which simplifies to

$$
\begin{aligned}
j(y, t) & =\operatorname{Re}\left[3 \sum_{n=-\infty}^{\infty}\left(\bar{N}_{A}^{n} \bar{I}_{A} e^{j(\omega t-n k y)}\right)\right]_{n=1,-5,7,-11,13, \ldots} \\
& =\left.0\right|_{n \neq 1,-5,7,-11,13, . .}
\end{aligned}
$$

This shows the well known MMF spatial series for a balanced 3-phase winding with backwards MMF wave for harmonic numbers 5, 11, 17 etc. and forwards rotating MMF for harmonic numbers 1, 7, 13, etc.. For a well designed winding it is often the case that only the fundamental MMF wave needs to be considered and we can obtain the per-phase equivalent circuit for this.

\subsection{Voltage equations}

If we only consider the fundamental forwards-rotating MMF wave for a balanced 3-phase machine then we can write the impedance matrix simply in terms of one phase current and one rotor current harmonic so that 


$$
\left[\begin{array}{l}
\bar{V}_{A} \\
0
\end{array}\right]=\left[\begin{array}{ll}
\bar{Z}_{s s} & \bar{Z}_{s r} \\
\bar{Z}_{r s} & \bar{Z}_{r r}
\end{array}\right]\left[\begin{array}{l}
\bar{I}_{A} \\
\bar{I}_{R}
\end{array}\right]
$$

which gives the voltage equations

$\bar{V}_{A}=\bar{Z}_{s s} \bar{I}_{A}+\bar{Z}_{s r} \bar{I}_{R}$

and

$$
0=\bar{Z}_{r s} \bar{I}_{A}+\bar{Z}_{r r} \bar{I}_{R}
$$

where

$$
\bar{I}_{R}=-\frac{\bar{Z}_{r s}}{\bar{Z}_{r r}} \bar{I}_{A}
$$

So that

$$
\bar{V}_{A}=\left\{\bar{Z}_{s s}+\frac{\bar{Z}_{s r}\left(-\bar{Z}_{r s}\right)}{\bar{Z}_{r r}}\right\} \bar{I}_{A}
$$

\subsection{Impedance components}

In (51) we can define

$$
\bar{Z}_{s s}=R_{A}+j X_{A_{-} \text {leak }}+j X_{s s}
$$

where $X_{A_{-} \text {leak }}$ is the stator winding leakage reactance which includes the end-winding leakage, slot leakage and differential leakage and $R_{A}$ is the phase resistance.

The main winding reactance is given by

$$
X_{s s}=3 \frac{2 \pi \omega_{s} r^{3} k_{s t k} L_{s t k} \mu_{0}}{k_{C}^{r} k_{C}^{s} g} \bar{N}_{A}^{1} \bar{N}_{A}^{-1}=3 X_{\text {coef }} \bar{N}_{A}^{1} \bar{N}_{A}^{-1}
$$

The stator-rotor impedance is

$$
\bar{Z}_{s r}=j X_{\text {coef }} k_{s k}^{1} \bar{N}_{A}^{1} \bar{N}_{r}^{-1} \times \text { Bar Number }=j X_{s r}
$$

And the rotor-stator impedance is

$$
\bar{Z}_{r s}=j 3 X_{\text {coef }} k_{s k}^{1} \bar{N}_{r}^{1} \bar{N}_{A}^{-1} \times \text { slip }=j X_{r s}
$$

In (19) the rotor differential is not denoted and can be calculated using standard expressions. However, we can use the conductor density method to calculated this where

$$
\begin{aligned}
\bar{Z}_{r r} & =2\left(1-\cos \left(n \theta_{b}\right)\right)\left(R_{b}^{1}+j X_{\text {slot }}^{1}\right)+2 R_{\text {end }- \text { ring }} \\
& +j 2 X_{\text {end-ring }}+j \sum_{\mu=-\infty}^{\infty} \frac{s X_{\text {coeff }}}{\left(1+\mu N_{b}\right)^{2}} \bar{N}_{r}^{\mu} \bar{N}_{r}^{-\mu} N_{b}
\end{aligned}
$$

Where $\mu=\ldots,-2,0,1, \ldots$ and the $\mu=0$ term is the main rotor rotor reactance $X_{r}$ and the sum of the other terms is the differential. $N_{b}$ is the bar number. Hence we can separate the terms in the last term of (56) which splits it into the main rotor inductance and the differential inductance. This will help with the derivation of the skew reactance term later. Hence

$$
\begin{aligned}
\bar{Z}_{r r} & =2\left(1-\cos \left(n \theta_{b}\right)\right)\left(R_{b}^{1}+j X_{\text {slot }}^{1}\right)+2 R_{\text {end-ring }} \\
& +j 2 X_{\text {end-ring }}+j\left(X_{r}+X_{\text {diff }}\right) \\
& =R_{r}+j X_{\text {slot }+ \text { ring }}+j X_{r}+j X_{\text {diff }} \\
& =R_{r}+j X_{r-\text { leak }}+j X_{r}
\end{aligned}
$$

The reactances in (57) do contain slip s terms.

\subsection{Equivalent circuit derivation}

Continuing to break down the voltage equation in (52) using (53)
$\bar{V}_{A}=\left\{R_{A}+j X_{A}++\frac{j X_{s s} \bar{Z}_{r r}-j X_{s r} j X_{r s}}{\bar{Z}_{r r}}\right\} \bar{I}_{A}$

so that

$$
\bar{V}_{A}=\left\{R_{A}+j X_{A}+\frac{j X_{s s}\left(\bar{Z}_{r r}-\frac{j X_{s r} j X_{r s}}{j X_{s s}}\right)}{\frac{j X_{s r} j X_{r s}}{j X_{s s}}+\left(\bar{Z}_{r r}-\frac{j X_{s r} j X_{r s}}{j X_{s s}}\right)}\right\} \bar{I}_{A}
$$

Hence

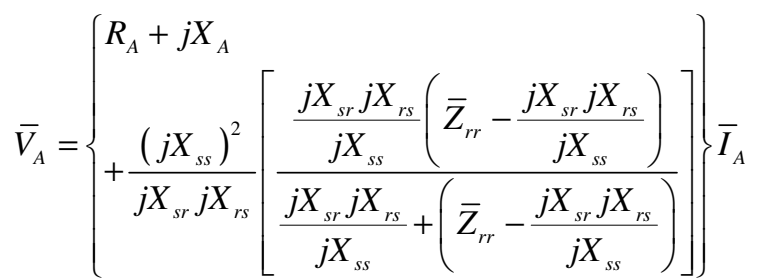

and further manipulation gives

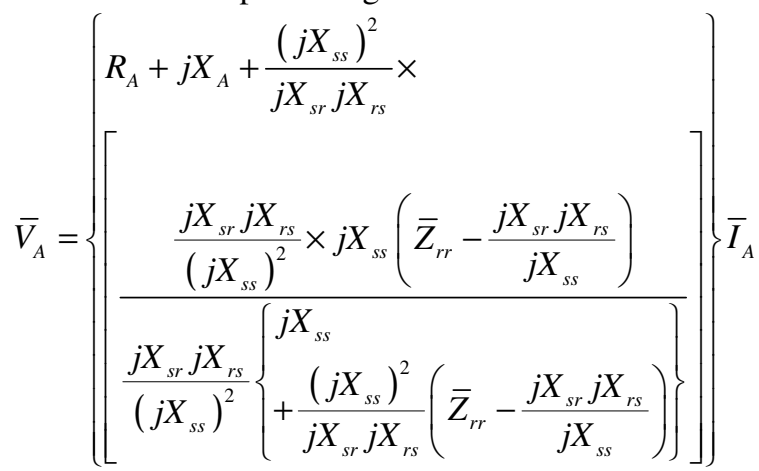

So that we can now simplify to

$$
\bar{V}_{A}=\left\{\begin{array}{l}
R_{A}+j X_{A}+\frac{\left(j X_{s s}\right)^{2}}{j X_{s r} j X_{r s}} \times \\
{\left[\begin{array}{c}
j X_{s s}\left(\bar{Z}_{r r}-\frac{j X_{s r} j X_{r s}}{j X_{s s}}\right) \\
\left\{j X_{s s}+\frac{\left(j X_{s s}\right)^{2}}{j X_{s r} j X_{r s}}\left(\bar{Z}_{r r}-\frac{j X_{s r} j X_{r s}}{j X_{s s}}\right)\right\}
\end{array}\right.}
\end{array}\right\} \bar{I}_{A}
$$

Equations (58) to (62) show the manipulations necessary to obtain an expression that can be related to the per-phase equivalent circuit. In this circuit the rotor components are referred to the stator, in (62) we can define a turns-ratio squared term between the stator and rotor circuits where

$\frac{\left(j X_{s s}\right)^{2}}{j X_{s r} j X_{r s}}=\left(\frac{n_{\text {stator }}}{n_{\text {rotor }}}\right)^{2}=\frac{3 \bar{N}_{A}^{1} \bar{N}_{A}^{-1}}{N_{b} k_{s k}^{2} \bar{N}_{r}^{1} \bar{N}_{r}^{-1} \times \text { slip }}=\frac{N^{2}}{s k_{s k}^{2}}$

Using (53) to (57) and (63), and after much further manipulation, the rotor impedance becomes

$$
\begin{aligned}
& \frac{\left(j X_{s s}\right)^{2}}{j X_{s r} j X_{r s}}\left(\bar{Z}_{r r}-\frac{j X_{s r} j X_{r s}}{j X_{s s}}\right) \\
& =\frac{R_{r}^{\prime}}{s k_{s k}^{2}}+\frac{j X_{r-\text { leak }}^{\prime}}{k_{s k}^{2}}+j X_{s s} \frac{\left(1-k_{s k}^{2}\right)}{k_{s k}^{2}}
\end{aligned}
$$

where

$R_{r}^{\prime} / s+j X_{r-\text { leak }}^{\prime}=N^{2} / s\left(R_{r}+j X_{r-\text { leak }}\right)$ 
The first term in (64) is the referred rotor resistance, the second term is the referred rotor leakage reactance and the third term is called the rotor skew reactance term. Typically the skew term from (5) is close to unity so that the skew reactance term will be much lower than $X_{s s}$ and if the skew is removed then the skew coefficient becomes unity and the skew reactance disappears. The equivalent circuit is then given in Fig. 5 and this is mathematically rigorous as described above. The core loss resistance is also included for completeness. However there are several variations of this which include a skew term in the magnetizing reactance $X_{s s}$ and variations in the skew reactance term with it split often between the stator and rotor circuits. The variation in skew reactance may be due to the fact that inter-bar current will reduce the skew reactance term and investigators have used empirical formulae to overcome this without including inter-bar currents (as discussed by Dorrell et al, 2003).

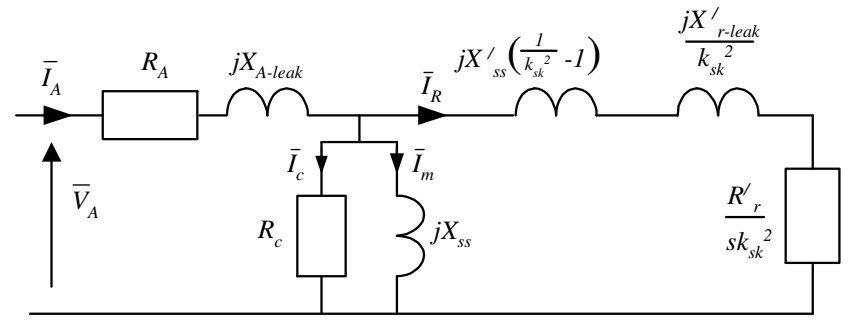

Figure 5 Equivalent circuit as derived

One variation in the equivalent circuit is given by Veinott, 1959, and this is shown in Fig. 6. This is a very common equivalent circuit and uses the same skew factor coefficient. If the rotor leakage and resistance are short-circuited then the input reactances are the same and equal to $\left(1-k_{s k}^{2}\right)$. For the small 3-phase machine in Dorrell, 2003, the machine has one stator slot skew and a skew factor $k_{s k}=0.994$. From Fig. 5 the skew reactance term in the rotor circuit is $3.35 \Omega$ when the magnetizing reactance is $259 \Omega$. For the circuit in Fig. 6, the total sum of the stator and rotor skew reactance is $2 \times 1.66=3.32 \Omega$. The division of the rotor impedances in Fig. 5 by $k_{s k}^{2}$ and the magnetizing reactance in Fig. 6 by $k_{s k}$ should have marginal effects since it is close to unity, therefore these circuits are likely to give similar results.

As already mentioned, Dorrell, 2003 did find that the skew reactance does seem to be overestimated due to interbar current and many authors use an attenuated value for the skew reactance or even miss it out completely.

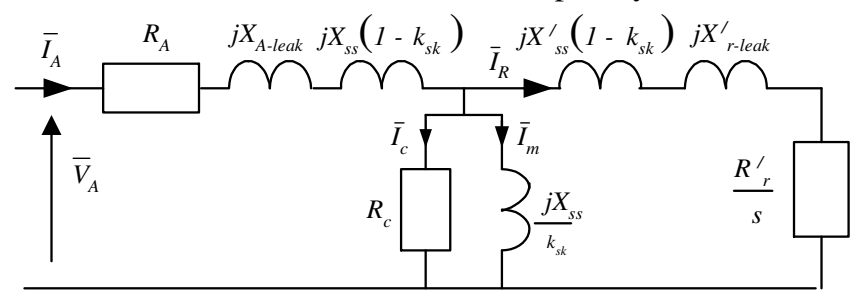

Figure 6 Equivalent circuit as used by Veinott

\section{CONCLUSIONS}

This paper has briefly discussed some of the different applications of the matrix analysis method then applied it to a split-phase machine in order to calculate the asynchronous torques in a machine with high winding harmonic. It was successfully applied which illustrates the strength and versatility of the method. The method was then used to derive the standard 3-phase induction motor equivalent circuit including the skew reactance term.

These algorithms are easily implementable in any software package (here the split-phase algorithm was coded in MATLAB) and represent the most modern form of induction machine analytical analysis and offers a level of sophistication above the more traditional per-phase analysis.

\section{REFERENCES}

S. Williamson and A. C. Smith (1982). Steady-State Analysis of 3Phase Cage Motors with Rotor-Bar and End-Ring Faults. Proc IEE, Part B, Vol 129, 1982.

S. Williamson and K. Mirzoian (1985). Analysis of Cage Induction Motors with Stator Winding Faults. IEEE PAS Transactions, Vol PAS-104, 1985.

S. Williamson, M. A. S. Abdel-Magied and A. C. Smith (1987). Steady-State Analysis of Double-Cage Induction Motors with Rotor-Cage Faults. Proc IEE, Part B, Vol 134, 1987.

D. G. Dorrell and A. C. Smith (1996). The calculation and measurement of unbalanced magnetic pull in cage induction motors with eccentric rotors. Part 1: analytical model and Part 2: Experimental investigation. Proc. IEE Electric Power Applications, Vol. 143, 1996.

D. G. Dorrell, TJE Miller and C. B. Rasmussan (2003). Interbar Current in Induction Machines. IEEE Transactions on Industry Applications, Vol 39, No 3, pp 677 - 684, 2003.

S. Williamson, A. C. Ferreira and A. K. Wallace (1997). Generalised theory of the brushless doubly-fed machine. Part I: Analysis. Proc. IEE Electric Power Applications, Vol. 144, 1997.

D. G. Dorrell (2007). Design Requirements for Doubly-Fed Reluctance Generators. IEEE PEDS Conference, Bangkok, Thailand, Dec 2007.

D. G. Dorrell and F. Jensen (2007). Modeling Split Phase Induction Motors with Center-Tapped Windings and Asynchronous Torque Dips. IEEE IAS annual meeting, New Orleans, Sept. 2007.

T.J.E. Miller (2006). SPEED's Electrical Motors. SPEED Laboratory, University of Glasgow, 2006.

C. G. Veinott (1959), Theory and Design of Small Induction Motors, McGraw-Hill Book Company, 1959.

\section{Biographical notes:}

D G Dorrell obtained his PhD from The University of Cambridge, UK, in 1993. He has held lecturing positions with The Robert Gordon University, UK and The University of Reading, UK. He is currently a Senior Lecturer with The University of Glasgow, a post he has held since 2001. He is also an Adjunct Associate Professor with the National Cheng Kung University, Taiwan and has visited several other institutes in recent years. His research areas cover electrical machine design and analysis and also renewable energy applications. He is a Chartered Engineer in the UK and a Fellow of the IET, London. He is also a Senior Member of the IEEE, USA. 